\title{
p38 and STAT3 activation by vGPCR in KSHV-infected cells
}

\author{
This article was published in the following Dove Press journal: \\ Virus Adaptation and Treatment \\ 2 September 2010 \\ Number of times this article has been viewed
}

Mingli Liu

Shanchun Guo

Sylvester Comprehensive Cancer Center, University of Miami Miller School of Medicine, Miami, FL, USA

Correspondence: Mingli Liu

Department of Microbiology, Immunology and Biochemistry, Morehouse

School of Medicine, 720 Westview

Drive SW, Atlanta, GA 303।0, USA

Tel +I 404752 I850

Fax + I 404752 II79

Email mliu@msm.edu
Abstract: The molecular mechanism whereby viral G protein-coupled receptor (vGPCR) signaling regulates vascular endothelial growth factor (VEGF) expression in Kaposi sarcoma (KS) formation remains incompletely defined. mECK36 cells, generated by transfection of mouse bone marrow endothelial cells with Kaposi's sarcoma-associated herpesviruses (KSHV) bacterial artificial chromosome (KSHVBac36), have been reported to be angiogenic, tumorigenic, and suitable for demonstrating a nonredundant role for vGPCR in KSHV-mediated tumorigenesis. In this report we used mECK36 and the cells composed of wild-type KSHVBac36 or the cells without vGPCR, namely vGPCR-null KSHVBac36 mutant, to dissect the molecular mechanisms of VEGF secretion induced by VGPCR in the context of KSHV infection. We found that VGPCR activates VEGF transcription via p38 MAPK and STAT3 in mECK36 and mECK36-derived cell models. We also found that in cells containing KSHV genome, STAT3 is tyrosine-phosphorylated and translocated into the nucleus, transactivating the target VEGF gene by binding to the specific DNA element TT $\left(\mathrm{N}_{4-5}\right)$ AA in a strictly vGPCR-dependent manner. Moreover, treatment of mECK36-derived cells with AG490 or a dominant negative STAT3 DNA vector showed strong inhibitory effects on vGPCR-induced VEGF promoter activity. In addition, vGPCR can upregulate STAT3 mRNA levels. Taken together, our findings show that vGPCR plays a nonredundant role in STAT3 activation in KSHV infected cells and that this activation plays an important role in the connection of the viral oncogene vGPCR and VEGF upregulation. Our results indicate the broad signaling activating capacity of vGPCR in the context of KSHV infection and suggest that the STAT3 pathway could be targeted for preventing KSHV-mediated angiogenesis in KS.

Keywords: Kaposi's sarcoma, vGPCR, p38, STAT3, KSHV

The DNA virus Kaposi's sarcoma-associated herpesviruses (KSHV), or a human herpesvirus 8 (HHV-8), a gamma-2 herpesvirus, was discovered in $1994 .{ }^{1-2} \mathrm{KSHV}$ is a causative agent for Kaposi's sarcoma, also associated with two other lymphoproliferative diseases, primary effusion lymphoma (PEL) and multicentric Castleman's disease. ${ }^{3}$ The KSHV genome contains approximately 90 open reading frame (ORFs) ${ }^{1,4}$ and encodes several viral oncogenes. Some of these viral oncogenes are latent genes, such as latency-associated nuclear antigen (LANA), v-cyclin $\mathrm{K},{ }^{2}$ viral Fas-associated death domain interleukin-1converting enzyme inhibitory protein (v-FLIP), viral interferon regulatory factor (IRFs, K10), and kaposin (K12). ${ }^{3}$ Some others are lytic genes, such as bcl-2, K1, vIL-6 (K2), and viral G protein-coupled receptor (VGPCR) gene. ${ }^{5}$ Although latent proteins are thought to play a critical role in KSHV-induced sarcoma, some observations still support the idea that latent infections alone are not sufficient to initiate and progress the sarcomagenesis. ${ }^{6}$ Instead, there is increasing evidence that vGPCR gene, which is the product of ORF74 of KSHV, could initiate Kaposi's sarcoma process and play a nonredundant role in 
KSHV-induced tumor development. ${ }^{6-8}$ Many research groups have documented the mechanisms by which vGPCR causes KS tumorigenesis, which can be summarized as follows: 1) expression of vGPCR directly transforms endothelial and fibroblast cells in vitro ${ }^{9-10}$ 2) deregulation of the viral gene program leads to nonlytic vGPCR expression; ${ }^{6}$ 3) vGPCR may delay or abort KSHV replication through p53-independent, p21mediated cycle arrest which inhibit CDK2 and provide vGPCR the necessary time to perform the proliferative and angiogenic functions, ${ }^{11}$ 4) paracrine cytokines from vGPCR-expressing cells activate transcription factors, such as NF- $\kappa B$ and NF-AT to induce VEGF expression or the KS-like lesions; ${ }^{2,6} 5$ ) autocrine mechanism by which autocrine factors from vGPCR-expressing cells generate proliferation and tumorigenesis directly;,2,12 6) Finally, some cofactors such as human immunodeficiency virus (HIV)-1 Tat, inflammation and aborted lytic cycle progression trigger the dysregulation of vGPCR. ${ }^{6}$

It has been demonstrated that the expression of this viral receptor could regulate a number of transduction pathways such as activation of phospholipase C, PKC, PI3-K/Akt/ GSK-3, TSC/mTOR, p42/44 MAPK, JNK/SAPK, and p38 MAPK as well as inhibition of adenyl cyclase. The downstream effectors transactivate specific transcription factors such as AP-1, NF- $\mathrm{B}, \mathrm{NF}-\mathrm{AT}$, and CREB which induce the target genes expression contributing to pathogenesis of KSHV. ${ }^{9,12-15}$ vGPCR also has been reported to target the Notch pathway by way of the transcription factor RBP-J ${ }^{11}$ and to activate the Heme oxygenase system leading to KS tumorigenesis. ${ }^{16}$ However, the relationship between vGPCR and STAT3, which is very important in the induction of cell transformation, angiogenesis, and tumorigenesis has rarely been examined. So far, only Burger et al reported that vGPCR can activate STAT3 and lead to neoplastic transformation in fibroblasts and endothelial cells. ${ }^{17}$ Based on this and the fact that constitutive STAT3 activity up-regulates VEGF expression and tumor angiogenesis, ${ }^{18}$ we assume that STAT3 may also be induced by vGPCR and mediate the vGPCR-derived angiogenesis by up-regulation of VEGF expression in mECK-derived cells. To test this hypothesis, we used the mECK36-derived cell models to investigate the role of STAT3 in vGPCR-induced VEGF expression.

\section{Materials and methods DNA constructs}

The vector pSilencer 2.1-U6 neo (SiRNA-control), SiRNA-vGPCR, pCEFLHA-vGPCR (vGPCR), or pCEFLHA vector were as described previously. ${ }^{1,10}$ pSTAT3-TA-Luc was purchased from Clontech Laboratories Inc. (Mountain view, CA). The DN-STAT3 and MSCV plasmids were gifts from Dr. Daniel C Link from the Department of Internal Medicine, Washington University School of Medicine, St. Louis, Missouri.

\section{Cells and transfection}

mECK36 cells were gifted by Dr. Enrique Mesri from the Department of Microbiology and Immunology and maintained as previously described. ${ }^{1}$ Briefly, mouse bone marrow endothelial cells (mEC) were obtained from Balb/C An Ncr-nu mice (NCI, Bethesda, MD) bone marrow. Mice femurs were flushed twice with phosphate-buffered saline (PBS), and the elutes were incubated in Dulbecco's modified Eagle's medium (DMEM) media plus 30\% fetal borine serum (FBS) (Gemini Bioproducts, Calabasas, CA), endothelial growth factor (EGF) $0.2 \mathrm{mg} / \mathrm{mL}$ (Sigma, St. Louis, MO), endothelial cell growth factor supplement (ECGS) $0.2 \mathrm{mg} / \mathrm{mL}$ (Sigma), heparin $1.2 \mathrm{mg} / \mathrm{L}$ (Sigma), insulin transferrine selenium (Invitrogen, Carlsbad, CA), penicilin-streptomicin 1\% (Invitrogen), and BME vitamin (VWR Scientific, Rochester, NY). KSHVBac36 was constructed by inserting a full-length recombinant KSHV genome into bacterial artificial chromosome, KSHVBac36 was stably transfected into $\mathrm{mEC}$ cells to generate mECK36 cells using lipofectamine 2000 (Invitrogen) and selected with hygromycin-B. The cells were then grown in the absence of hygromycin to negative select cells and therefore generate mECK36-KSHV-Null cells, which lost the KSHV episome (KSHV episome was measured by GFP marker). Next, KSHVBac36 construct was retransfected into mECK36-KSHV-Null cells to generate BBac36. Finally, the genotypic markers of vGPCR were knocked out from KSHVBac36 by transposon mutagenesis to generate ORF74/vGPCR deletion mutant and stably transfected into mECK36-KSHV-Null cells to create B $\Delta$ vGPCR cells in the presence of hygromycin selection.

\section{Luciferase reporter gene assay}

The STAT3 reporter construct (pSTAT3-TA-Luc) expresses the firefly luciferase gene under the regulation of TA minimal promoter containing four tandem binding sites for STAT3. pGL3-VEGF Luc is a reporter plasmid, which expresses the luciferase gene under the control of the full-length VEGF promoter; whereas pst pGL3-VEGF Luc, is a truncated VEGF promoter construct lacking the HRE (HIF-responsive element). ${ }^{12}$ mECK36-derived cells were transfected using lipofectamine 2000 (Invitrogen) with $0.5 \mu \mathrm{g}$ of luciferase reporter construct, different amounts of expression plasmids together with $100 \mu \mathrm{g}$ of pRL-TK, a cytomegalovirus-Renilla 
vector to control transfection efficiency. The amount of total DNA transfected was equalized with the appropriate amounts of control vectors. After transfection at different indicated time points, cells were harvested and lysed in reporter lysis buffer (Promega, Madison, WI). Luciferase activity was determined by using the Dual Luciferase Kit (Promega) and a luminometer (Turner Design, Sunnyvale, CA) according to the manufacturer's recommendation. All luciferase results were normalized to Renilla activity from the cotransfected pRL-TK plasmid. The data for luciferase activity are expressed as the fold induction with respect to control cells and are the mean \pm standard error of triplicate samples.

\section{VEGF quantization by ELISA}

$\mathrm{B} \triangle \mathrm{vGPCR}$ and BBac36 cells were seeded at $5 \times 10^{4}$ cells/mL in regular growth medium for 72 hours. Supernatants were collected by centrifuging cells at $800 \mathrm{rpm}$ for 2 minutes, and cell counts were taken to ensure consistency in different wells. VEGF in supernatants was quantified by using commercial enzyme-linked immunosorbent assay (ELISA) plates precoated with the purified polyclonal antibody specific for mouse VEGF (R\&D Systems Inc, Minneapolis, MN). The values of reading were normalized to cell numbers in each cell lines, and are depicted as picograms per milliliter per 1000 cells.

\section{Western blot analysis}

mECK36-derived cells were transfected with vGPCR or with empty vector using lipofectamine 2000 according to the manufacturer's instructions. Forty-eight hours post-transfection, the cells were washed with DPBS (Gibco, Carlsbad, CA) and were incubated with the serum-free medium X-VIVO10 (Cambrex, Baltimore, MD) for another 24 hours. Cells were then washed with cold PBS, and lysed in $50 \mathrm{mM}$ HEPES (pH 7.5), $150 \mathrm{mM}$ $\mathrm{NaCl}, 1.5 \mathrm{mM} \mathrm{MgCl}_{2}, 1 \mathrm{mM}$ EGTA, 10\% glycerol, 1\% NP-40-100 mM NaF, $10 \mathrm{mM}$ sodium pyrophosphate, $0.2 \mathrm{mM}$ sodium orthovanadate, $1 \mathrm{mM}$ phenylmethylsulfonyl fluoride, $10 \mu \mathrm{g}$ of aprotinin $/ \mathrm{ml}$. Aliquots of lysates were resolved by 4\%-15\% SDS-polyacrylamide gels and transferred to nitrocellulose membranes. They were probed with the appropriate antibodies and developed using the ECL system (Amersham Biosciences, Piscataway, NJ).

\section{Immunofluorescence staining}

mECK36-derived cells were plated on glass cover slips and transfected with pCEFLHA, vGPCR, SiRNA-control, and SiRNA-vGPCR as indicated with lipofectamine 2000. Cells were fixed in $4 \%$ paraformaldehyde for 10 minutes at room temperature, blocked with $10 \%$ goat serum and 1\% BSA; incubated with anti-phosphorylated STAT3 (Y705), or anti-HA primary antibodies for 1 hour; washed and then incubated with Alexa-555- or Alexa-647-conjugated secondary antibodies (Molecular Probes, Carlsbad, CA) for 1 hour. Cover slips were mounted onto slides with Vectashield mounting medium for fluorescence with DAPI (H-1200; Vector Laboratories Inc., Burlingame, CA). Fluorescent images were collected by using a Zeiss LSM510 confocal microscope; images were captured with LSM software version 2.3 (Carl Zeiss, Wetzlar, Germany).

\section{Real-time PCR and RT-PCR analysis}

Total RNA was isolated from cells using an RNeasy Mini Kit (Qiagen, Valencia, CA). cDNA was synthesized from the isolated RNA using Taqman Gold reverse transcription reagents (Applied Biosystems, Foster City, CA). Reverse transcription was performed by using random hexamers at $25^{\circ} \mathrm{C}$ for 10 minutes, $42^{\circ} \mathrm{C}$ for 60 minutes, and $95^{\circ} \mathrm{C}$ for 5 minutes. Quantitative PCR was performed using SYBR Green PCR Master Mix (Applied Biosystems, Warrington, UK) in a GeneAmp 7300 sequence detection machine (Applied Biosystems). The data were analyzed using ABI Prism 7700 Sequence Detection System (Applied Biosystems). Sequences of forward and reverse primers were as follows: VEGF 5' CAAGTCCGAATGCAGATCCT3'; 5'TGTCTGGCTTCACAGCACTC3'; STAT3 5'AGGACATCAGTGGCAAGACC 3'; 5'TGGTCGCATCCATGATCTTA3'. The glyceraldehydes3-phosphate dehydrogenase (GAPDH) housekeeping gene was amplified in parallel to all reactions to ensure that equal amounts of starting cDNA were used in each reaction. The primers for GAPDH were 5'AGAACATCATCCCTGCATCC $3{ }^{\prime}$ and 5'CACATtGgGgGtAGGAACAC3'. Amplifications for part-length mouse vGPCR were performed with 35 cycles of 30 seconds at $95^{\circ} \mathrm{C}, 30$ seconds at $60^{\circ} \mathrm{C}$, and 30 seconds at $72^{\circ} \mathrm{C}$. The PCR products were detected by electrophoresis in agarose gels and fluorescence under UV light upon ethidium bromide staining. The primers for vGPCR were 5'CTGCATGTCAGAACCGTGTC3' and 5'ACAGTGCCTGCAGTAACGAG3'. The primers for part length GAPDH were 5'CCACCCAGAAGACTGTGGAT3' and 5'ACCCTGTTGCTGTAGCCGTA3'.

\section{Antibodies and reagents}

The following antibodies were used: rabbit polyclonal antiphospho-STAT3 (Tyr705), rabbit polyclonal anti-total-STAT3, rabbit polyclonal anti-phospho-p38 MAP Kinase (Thr180/ Tyr182), rabbit polyclonal anti-total-p38 MAP Kinase, mouse monoclonal anti-HA-Tag (Cell Signaling, Danvers, MA), and 
rabbit polyclonal anti- $\beta$ actin (Sigma). The secondary antibodies were peroxidase goat anti-rabbit IgG (Oncogene Science Inc., Manhasset, NY), peroxidase goat anti-mouse IgG (Oncogene), Alexa Fluor ${ }^{\circledR} 555$ goat anti-rabbit IgG, and Alexa Fluor ${ }^{\circledR}$ 647 goat anti-mouse IgG (Molecular Probes). AG490 was purchased from BIOMOL Research Laboratories Inc (Plymouth Meeting, PA). SB203580 was purchased from Calbiochem (La Jolla, CA). Cobalt chloride was purchased from Sigma.

\section{Statistical analysis}

A Student's $t$-test was applied for the statistical determinations. $P<0.05$ was considered significant. All experiments were repeated at least three times.

\section{Results \\ vGPCR induces VEGF expression by transactivating VEGF promoter}

To test whether vGPCR activates VEGF transcription, we tested the effect of vGPCR on the expression VEGF both at mRNA and protein level. Firstly, we found that cell phenotypes vary in cells with vGPCR and without vGPCR in our cell models. Cells harboring vGPCR gene are typical spindleshaped as described by other groups, ${ }^{8,10,16}$ whereas cells with vGPCR gene knock-out have a flattened, round-up morphology (Figure 1A). Real-time quantitative PCR results showed that genetic knock-out of the vGPCR gene significantly decreases VEGF expression by one quarter $(P<0.05)$ (Figure $1 \mathrm{~B})$; reintroduction of $v G P C R$ into $B \triangle v G P C R$ cells resulted in restoration of VEGF mRNA expression in a dose-dependent manner (Figure 1C). Consistent with results from RNA level, secretion levels of VEGF in BBac36 was also proven to be 2.21-fold higher than B $\Delta$ vGPCR cells $(P<0.001)$ by ELISA (Figure 1D). We next performed a luciferase assay to attest the mechanism involving the activation of VEGF by vGPCR. Cells with or without vGPCR were transfected with VEGF luciferase reporter construct as well as internal control vector pRL-TK, a cytomegalovirus-Renilla vector to control transfection efficiency. The amount of total DNA transfected was equalized with the appropriate amounts of control vectors. Compared to B $\Delta \mathrm{vGPCR}$ cells, BBac36 cells showed a 2.59-fold increase in VEGF luciferase expression, and even as high as 5.21-fold in the presence of $\mathrm{CoCl} 2$ (Figure 1E). Figure 1E also indicated that truncated VEGF promoter construct lacking the HRE impaired transcription activity. All the above results support the findings that vGPCR may activate VEGF gene expression at the gene transcriptional level ${ }^{9,10}$ by other research groups previously. Our results also show that knock-down vGPCR by SiRNA-vGPCR decrease transcriptional activity of the VEGF promoter by $40 \%$ proportionally (Figure 1F, left) in BBac36 cells; conversely overexpressing vGPCR by cotransfected a vGPCR expression vector in $\mathrm{B} \triangle \mathrm{vGPCR}$ cells enhanced transcriptional activity of VEGF promoter in (Figures $1 \mathrm{~F}$ and $1 \mathrm{G}$ ), which indicates that vGPCR modifies VEGF transcription.

\section{vGPCR activates stress-induced kinase P38 MAP kinase}

We next sought to determine whether vGPCR activates p38 MAP kinase. A vGPCR expression vector was transfected into mECK36, BBac36, mECK36 KSHV-null, and $\mathrm{B} \triangle \mathrm{vGPCR}$ cells, respectively. Whole cell extracts of the different cells were analyzed by Western blotting with antibody to the phosphotyrosine residue within p38. Our data showed mECK36 and BBac36 cells express low level of phosphorylated p38 protein, and vGPCR increases level of phosphorylate $\mathrm{p} 38$ protein in all four mECK36-related cells (Figure 2A). These results are consistent with other groups' data in which vGPCR was found to up-regulate VEGF expression and secretion through the p38 MAP kinase pathway in NIH3T3 cells and in human umbilical vein endothelial cells HUVECs. ${ }^{9,12}$ Activation of p38 pathways have also been described as essential in KSHV infection, ${ }^{9,12}$ however the basal level of phosphorylated p38 in mECK36 and BBac36 cells in our data were relatively weak.

To assess whether p38 modifies VEGF transcriptional activity, we tested the effects of p38 inducers and inhibitors on the VEGF promoter activity. A vGPCR expression vector and either expression vector MEKK6 (a potent activator of p38) or MEKK6KR (a dominant negative MEKK6) were cotransfected along with VEGF luc and pRL-TK into BDvGPCR cells and BBack36 cells, respectively. MEKK6 potentially increased the transcriptional activity of VEGF promoter activity, as shown in Figure 2B. Expression of the MEKK6KR considerably reduces the VEGF promoter activity induced by vGPCR (Figure 2C). The effects of SB203580 (SB), a specific pharmalogical inhibitor of p38 MAP kinase were also investigated. As with MEKK6KR, VEGF promoter activity was inhibited by SB203580, whereas the control solvent did not inhibit its activity (Figure 2D).

\section{Activation of STAT3 signaling by vGPCR in mECK-derived cells}

Constitutive activation of STAT3 has been reported in several primary cancers and tumor cell lines, as well as in vGPCR 


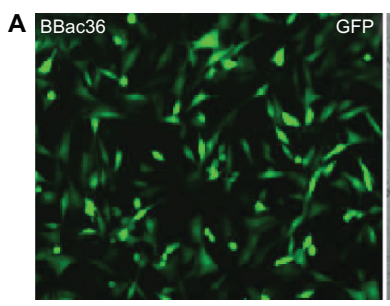

B

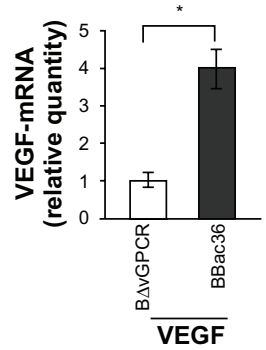

E

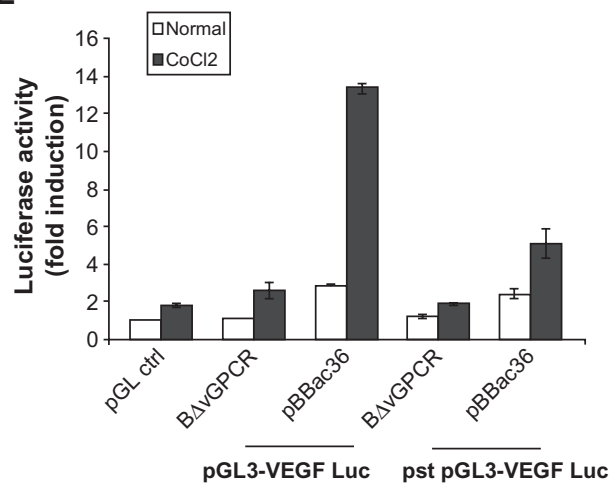

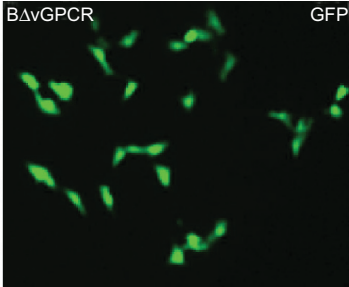

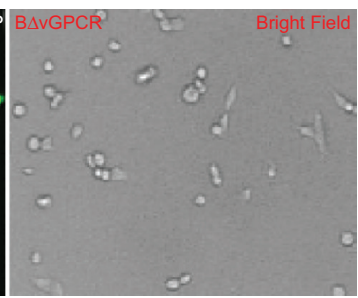

C

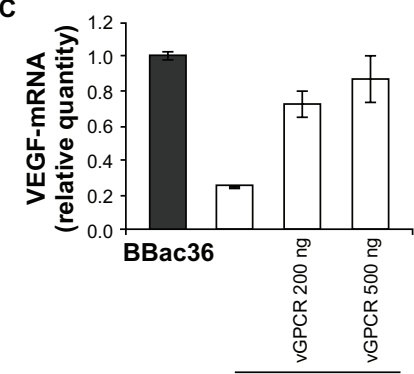

D

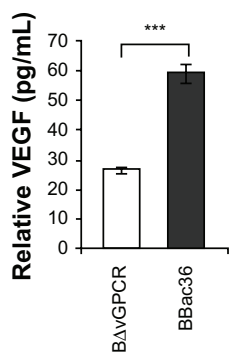

B $\triangle$ vGPCR

F
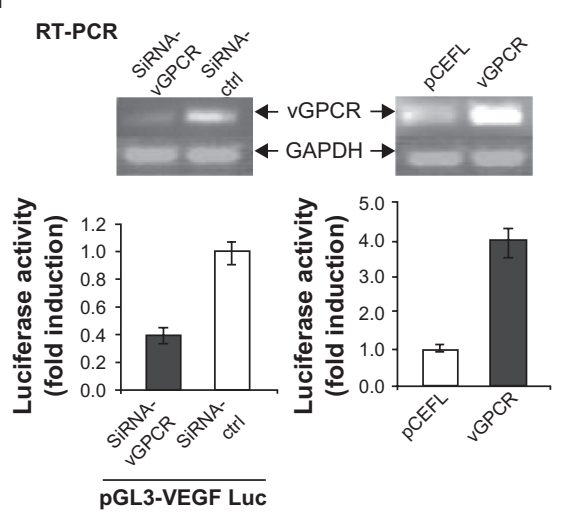

$\mathbf{G}$

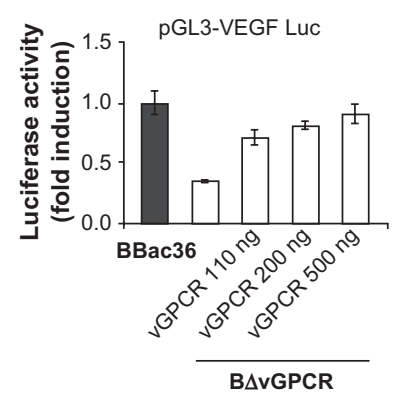

Figure I vGPCR inducesVEGF expression by activating transcription of VEGF promoter: $\mathbf{A})$ morphology of $B B a c 36$ and $B \Delta v G P C R$ cells in cultures (Original magnification, $\times 10)$. Cells expressing GFP indicated the presence of KSHV episome; B) vGPCR increases VEGF expression at mRNA levels. VEGF mRNA expressions in BBac36 and BAvGPCR cells were quantitated by real-time PCR, and relative quantities are represented on the $y$ axis. The data of three independent experiments performed in triplicates are shown; C) vGPCR overexpression increases VEGF mRNA level in a dose-dependent manner. B $\triangle \mathrm{vGPCR}$ cells were cotransfected with different amounts of vGPCR and empty vector, then total RNA was extracted and used to measured the VEGF mRNA levels as described in Figure IB; D) VEGF quantitation by ELISA. BBac36 and BAvGPCR cells were treated as described in Materials and Methods section. Results shown are averages of three independent samples with the standard deviations represented by error bars and the values are depicted as picograms per milliliter per 1000 cells; E) Induction of transcription from VEGF promoter in BBac 36 and B $\Delta$ vGPCR cells. BBac 36 and $B \Delta v G P C R$ cells were cotransfected with PGL3-VEGF luc, or pst PGL3-VEGF Luc, along with pRenilla-Luc per well as indicated. In some experiments, CoCl2 was added in media to give a final concentration of $100 \mu \mathrm{M}$. Twenty hours after transfection, lysates were assayed for luciferase activities. Luciferase activity was measured and normalized to the Renilla activity. Results are the mean \pm SE of three independent experiments performed in triplicate and expressed as the fold induction relative to appropriate control; $F$ ) SiRNA-vGPCR inhibits, overexpression of vGPCR activates the VEGF promoter. SiRNA-vGPCR, SiRNA-control, vGPCR, and pCEFLHA vectors where indicated were cotransfected into BBac36 cells. Total RNA were isolated from parallel plates, and vGPCR levels were detected by RT-PCR (upper bands of upper panel). GAPDH was used as an internal control to monitor the presence of amplified cDNA in the samples (lower bands of upper panel). Lower panels show the luciferase activity of BBac36 cells which were transfected with the different expression plasmids as indicated above, together with of pGL3-VEGF luc and of pRenilla; G) Reintroduction of the vGPCR gene into B $\triangle \mathrm{vGPCR}$ cells result in recovery of the VEGF promoter activities, furthermore vGPCR enhances VEGF promoter activity in a dose-dependent manner. 
A

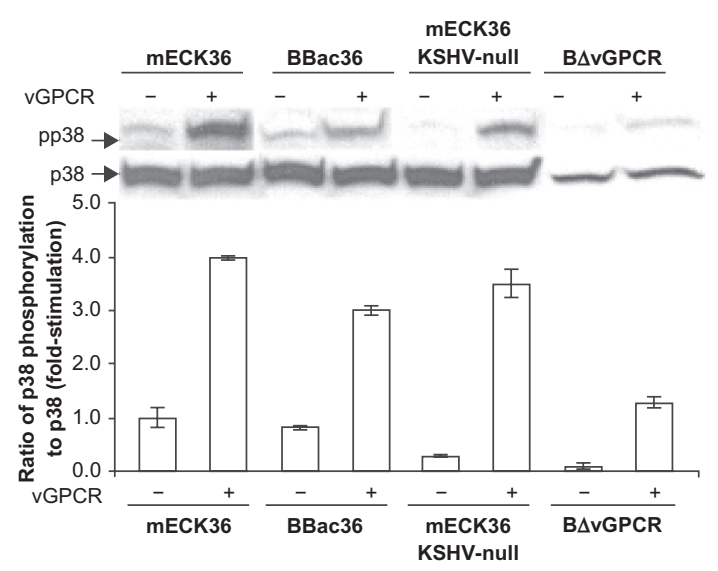

C

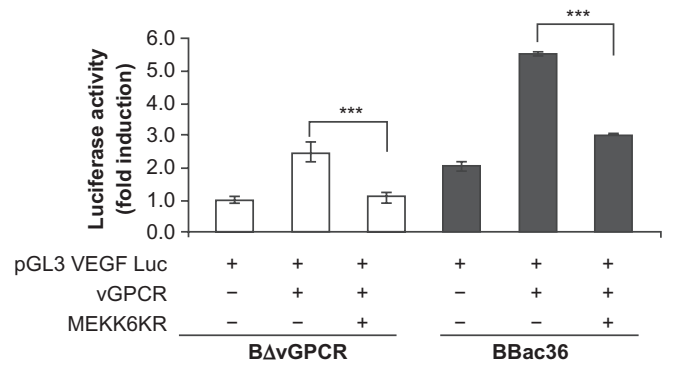

B

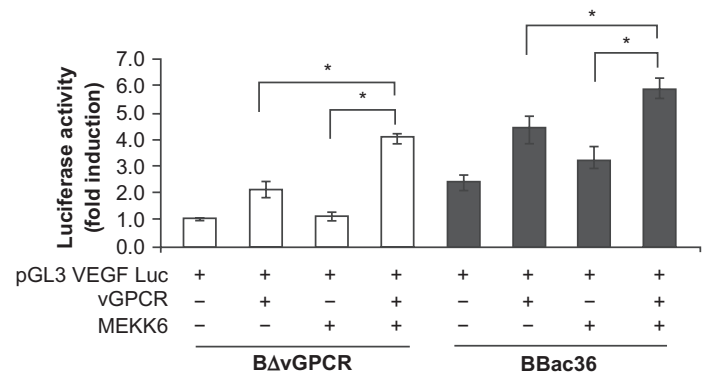

D

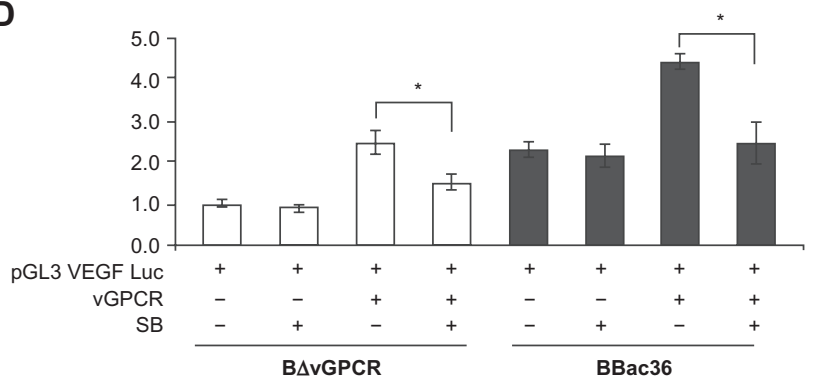

Figure 2 vGPCR activates stress-induced p38 MAP kinase; A mECK36-derived cells, mECK36, mECK36 KSHV-null, BBac36, and BAvGPCR cells were transfected by lipofectamine 2000 with vGPCR plasmid and empty vector, respectively. Forty-eight hours after transfection, cells were serum-starved for 12 hours. Then protein extracts were probed with antibodies to phosphorylated p38 MAP kinase (Thr I80/Tyr I82) and total p38 MAP Kinase. $\beta$-actin is used as an internal control to monitor protein amounts in each lane. The data represent one of three independent experiments with similar results. Beneath the Western blot is a densitometeric analysis of the bands performed with the ImageQuant program. The data are expressed as the fold stimulation relative to appropriate control cells and are the mean \pm SE of duplicate samples performed in three independent experiments; B to D B $\triangle \mathrm{vGPCR}$ and Bac36 cells were transiently transfected with the pGL3 VEGF Luc plasmid, vGPCR, together with pRanilla. Cotransfection with MEKK6 was as indicated in panel B, cotransfection with MEKK6KR was as indicated in left panel of C. SB203580 was added in media to give a final concentration of $10 \mu \mathrm{M}$ as showed in panel D. Twenty-four hours after transfection, cells were lysed and luciferase activity was measured and normalized to the Renilla activity.

transfected NIH 3T3 cells. ${ }^{17}$ We set out to examine whether the persistent activation of STAT3 is also present in mECK36derived cells. A vGPCR expression vector was transfected into mECK36 and mECK36 KSHV-null (Figure 3A) as well as $\mathrm{BBac} 36$ and $\mathrm{B} \Delta \mathrm{vGPCR}$ cells (Figure $3 \mathrm{~B}$ ), respectively. Whole cell extracts of the different cells were analyzed by Western blotting with antibody to the phosphotyrosine residue 705 within STAT3. As shown in Figures 3A and 3B, extracts of mECK 36 and BBac36 cells showed constitutive STAT3 phosphorylation at Tyr 705. In contrast, the mECK36 KSHV-null and B $\triangle \mathrm{vGPCR}$ cells showed very weak level of STAT3 phosphorylation. On average STAT3 phosphorylation increased 1.4-fold and 2.5-fold compared to respective parental cells. By overexpressing vGPCR in mECK36-related cells, the STAT3 phosphorylation level further increased 2.1-fold to 4.3-fold in the four mECK-related cells, respectively. Together, these results indicated that vGPCR indeed activates STAT3 by phosphorylation of STAT3. As we know, the typical activation of STAT3 is through phosphorylation on tyrosine residues in $\mathrm{C}$-terminal domains and then translocation to the nucleus, binding to specific promoter region of target genes and thereby regulating gene expression. ${ }^{19}$ For that reason, we next determined subcellular localization of STAT3 protein in our system. We used confocal microscopy to determine the localization of STAT3 in the absence or presence of vGPCR in $\mathrm{B} \Delta \mathrm{vGPCR}$ and BBac36 cells similar to those used for Figures 3A and 3B. Either a HA-tagged vGPCR expression vector or SiRNA-vGPCR were transfected into B $\triangle$ vGPCR and BBac36. pSTAT3 was mostly nuclear and with very little, or weak cytoplasmic staining for all mECK36-derived cells deprived of serum. This is representatively shown in Figures $3 \mathrm{C}$ and $3 \mathrm{~F}$. To gain quantitative analysis of pSTAT3 antibody immunoreactive signals in mECK36 versus mECK36 KSHV-null cells, we acquired fluorescence intensity for 15 cells from each cell line by using a MetaMorph Imaging System software program (version 3.68; Universal Imaging, West Chester, PA). The average relative intensity in nucleus was $119.04 \pm 11.33(n=15)$ in mECK36 cells. In contrast, for the mECK36 KSHV-null cultures, the average immunofluorescence intensity with pSTAT3 antibody in 
A
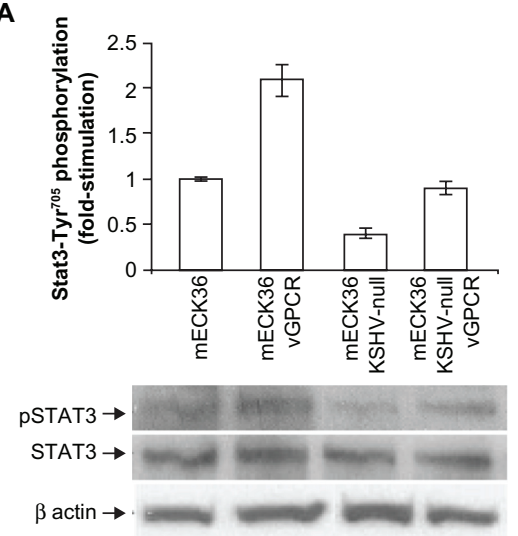

C

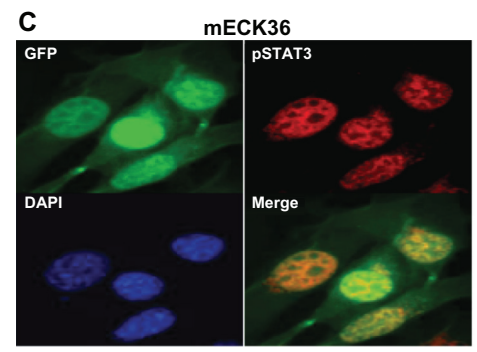

$\mathbf{F}$

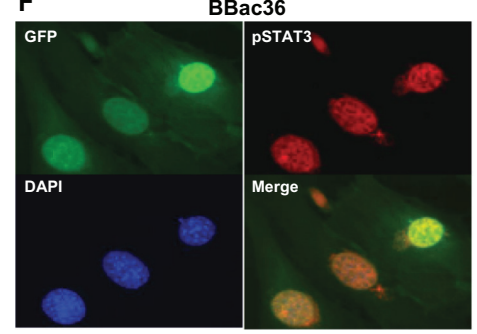

$\mathbf{J}$

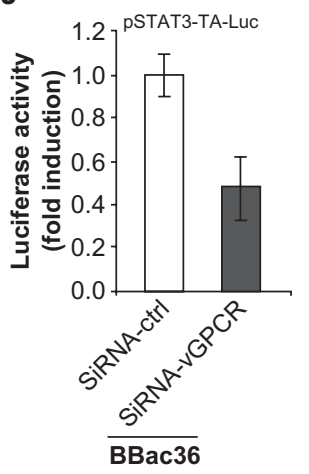

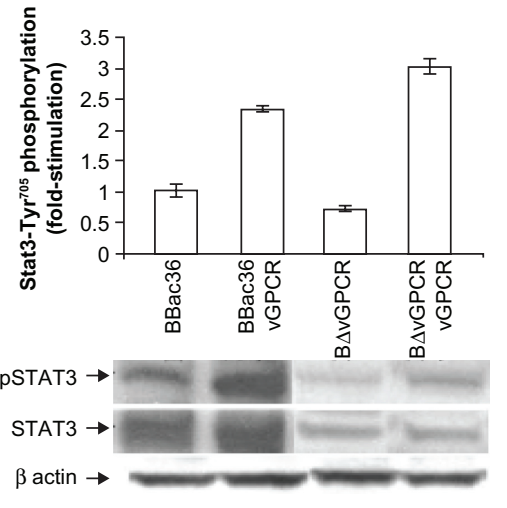

E

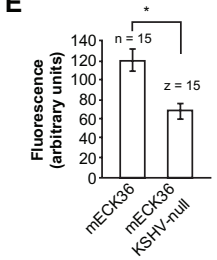

G BAVGPCR

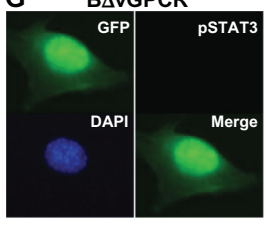

I BAVGPCR.vGPCR

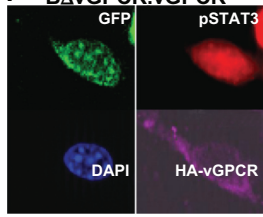

K

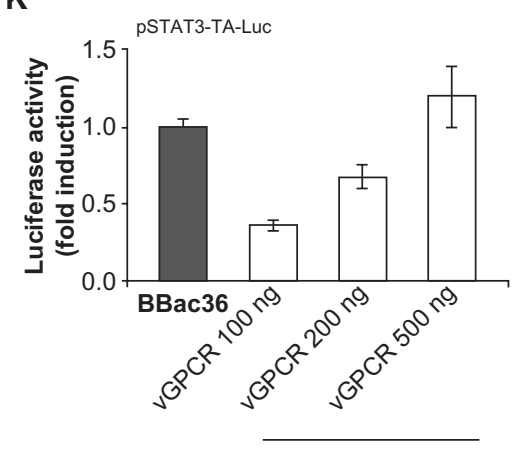

Figure 3 Activation of STAT3 signaling by vGPCR in mECK36-derived cells; $\mathbf{A}$ and B STAT3 is persistently activated in mECK36-derived cells. mECK36, mECK36 KSHV-null, $\mathrm{BBac} 36$, and B $\triangle \mathrm{vGPCR}$ cells were treated as in Figure 2. The cell lysates were probed with antibodies to phosphorylated STAT3 (Y705) and total STAT3. $\beta$-actin is used as an internal control to monitor protein amounts in each lane (lower panel). The data represent one of three independent experiments with similar results. Above the Western blot is a densitometeric analysis of the bands performed with the ImageQuant program (upper panel). The data are expressed as the fold stimulation relative to appropriate control cells and are the mean \pm SE of duplicate samples performed in three independent experiments; C-I Expression of vGPCR leads to the phosphorylation of STAT3 and induces its translocation to the nucleolus. Panels C and D: mECK36 and mECK36 KSHV-null cells were treated as described in Materials and Methods section. GFP-positive cells indicated KSHV infection. mECK36 KSHV-null lost GFP fluorescence. Panel E: Quantitation of nuclear phosphorylated STAT3 staining from samples of mECK36 and mECK36 KSHV-null cells. Total nuclear immunofluorescence intensity with PSTAT3 antibody (red) was determined with MetaMorph Imaging System software program (version 3.68; Universal Imaging, West Chester PA), and the average value for the indicated number of cells examined is shown. The asterisks indicate significant difference with a $p$ value of $<0.05$ as determined with Student's paired $t$-test. Panels F to I: BBac36 and B $\triangle \mathrm{vGPCR}$ cells were treated with SiRNA-vGPCR, vGPCR, and empty vector, respectively, using the same methods as described in panels A and B, and cells were also stained for HA-vGPCR with an anti-HA monoclonal antibody J and $\mathbf{K}$ Extracts from vGPCR-expressing cells activate a GAS-dependent promoter driving luciferase expression, STAT3 DNA-binding activity was reduced in cells with vGPCR-knock out or vGPCR knock-down. BBac36 cells and $B \triangle v G P C R$ cells cotransfected with SiRNA-vGPCR, SiRNA-control, different amounts of vGPCR, PCEFLHA empty vector as indicated, together with pSTAT3-TA-Luc. 
nucleus was 68.31 $\pm 7(P<0.05)$ (Figures 3C, 3D, and 3E). The amount of nuclear phosphorylated STAT3 in BBac36 cells was similar to that in mECK36 cells (Figure 3F). It is not surprising that pSTAT3 was hardly detected in nucleus when gene vGPCR was genetically knocked out from BBac36 cell (Figure $3 \mathrm{G}$ ). Similarly, phosphorylated STAT3 failed to undergo nuclear accumulation in cells with vGPCR mRNA knockdown mutants, and was shown with very little cytoplasmic staining in Figure 3H. As we expected, reintroduction of vGPCR gene into $\mathrm{B} \triangle \mathrm{vGPCR}$ cells resulted in restoration of the nuclear accumulation of phosphorylated STAT3 (Figure 3I). Taken together, these results suggest that vGPCR is essential for pSTAT3 to accumulate in the nucleus in mECK36-related cells. Moreover, the observations by confocal microscopy that the basal levels of nuclear phosphorylated STAT3 were very low in mECK $36 \mathrm{KSHV}$-null and $\mathrm{B} \Delta \mathrm{vGPCR}$ cells agree with our results by Western immunoblotting. To test the functional interaction between vGPCR and STAT3, we examined whether vGPCR affects STAT3 mediated transcriptional activation. The induction of STAT3 was monitored using pSTAT3TA-Luc, which contains four copies of the STAT3 GAS-like binding sites. ${ }^{20}$ The measurement of STAT3 DNA binding activity to STAT3 specific sequences showed that STAT3 luc activity decrease about one half in cells with vGPCR mRNA knock-down (left panel of Figure 3J) whereas ectopically expressing vGPCR in B $\Delta \mathrm{vGPCR}$ and BBace 36 cells cause an increase in luciferase activity of about 3.30-fold and 13.36fold, respectively (right panel of Figure 3J). Based on this, we evaluated the effect of transient transfection of different amounts of vGPCR on the GAS-dependent promoter activity and found increasing concentration of vGPCR steadily enhanced the GAS-dependent promoter activity. The phenomenon that pSTAT3-TA-Luc responded to vGPCR overexpression in a dose-dependent manner indicated that vGPCR was able to affect the STAT3 DNA binding activity to a target gene promoter (Figure 3K).

\section{STAT3 upregulates VEGF expression through the VEGF gene promoter}

We further examined whether the VEGF promoter is regulated by STAT3. To determine the influence of JAK2-STAT3 signaling on VEGF expression, we tested VEGF promoter activity in transfected $\mathrm{B} \Delta \mathrm{vGPCR}$ and $\mathrm{BBac} 36$ cells in response to JAK2 inhibitor AG490. As expected, luciferase reporter assay revealed that the activity of pGL3 VEGF promoter (containing two putative STAT3-binding sites) induced by vGPCR was inhibited in transfected B $\triangle \mathrm{vGPCR}$ and BBac36 cells about 3.18-fold and 2.12-fold respectively in the presence of AG490
(Figure 4A, left panel). The right panel of Figure 4A shows the Western blotting results of AG490 inducing down-regulation in pSTAT3 without significant changes in STAT3 level. To further rule out secondary effects of AG490, we assumed that blocking the expression of STAT3 protein should have the same effects as that of inhibiting action of AG490. Indeed, VEGF promoter was not inactivated by MSCV empty vector. Notably, expression of dominant negative STAT3 considerably reduced the VEGF promoter activity induced by vGPCR (Figure 4B). These observations suggest that STAT3 mediates vGPCRstimulated VEGF expression and that the VEGF promoter could be directly regulated by STAT3 at the transcriptional level.

\section{STAT3 mRNA level was elevated by VGPCR}

Since two potential STAT3-binding sites TT $\left(\mathrm{N}_{4-5}\right)$ AA were found in STAT3 promoter using the TFSEARCH program, we asked whether STAT3 can be transactivated by itself. To test this hypothesis, we examined the mRNA expression of STAT3 in B $\triangle \mathrm{vGPCR}$ and BBac36 cells transfected with increasing amounts of vGPCR vector using real-time RTPCR. We found that mRNA levels of STAT3 were elevated as vGPCR concentrations were increased (Figure 5). It could be reasonably inferred that activated STAT3 after stimulation by vGPCR enters into the nucleus and binds to STAT3 specific DNA elements of its own, activating the transcription expression of STAT3 itself.

\section{Discussion}

As it was proposed that spindle cells of Kaposi's sarcoma lesions originated from endothelial cells (vascular endothelial cells or lymphatic endothelial cells), several models of endothelial cells infected with KSHV have been used to elucidate the mechanism of Kaposi sarcomagenesis. ${ }^{21-24}$ The advantages of using these cell models are obvious, eg, the KSHV-infected cells may express KS spindle cell markers and have the transformation ability, which is indispensable for the KS-derived tumor development. Compared to previous models, the mECK6 model exhibits evidence of the characteristic angiogenenic and tumorigenic response of the KSHV infected cells in KS lesions. ${ }^{1}$

Although vGPCR has been shown to induce a KSphenotype by upregulation of VEGF, ${ }^{2,7-10}$ the mechanism by which viral oncogene vGPCR signals downstream to VEGF is not well understood. Since several tumor viruses genes are linked to STAT3 activation, it was expected that STAT3 
A

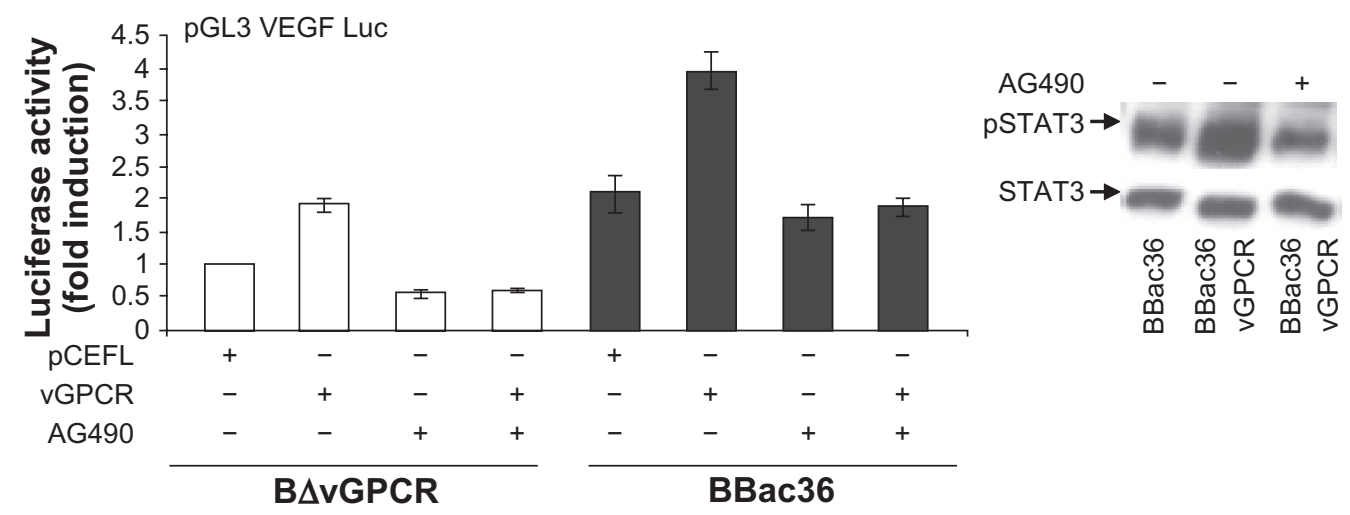

B

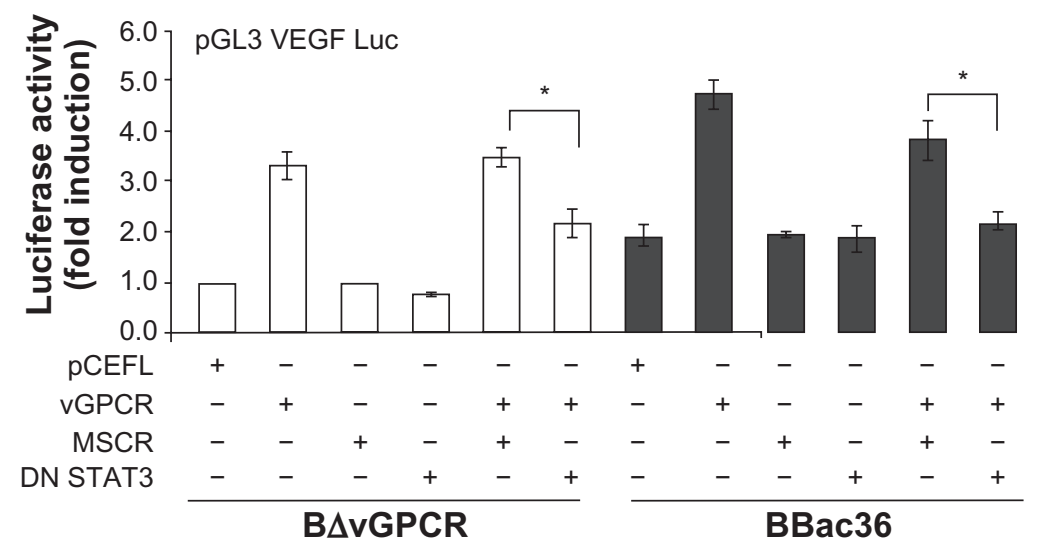

Figure 4 STAT3 up-regulates VEGF expression through the VEGF gene promoter; A Left panel: B $\triangle \mathrm{vGPCR}$ and BBac36 cells were transiently transfected with of pGL3 VEGF Luc along with of pRenilla-Luc. Cotransfection with vGPCR and empty vector were as indicated. Forty-eight hours after transfection, cells were serum starved for 12 hours. One hour before cell harvest, AG490 was added in media to give a final concentration of $50 \mu \mathrm{M}$. An identical volume of DMSO was added as a control to rule out inhibitory effects of the solvent of AG490. Right panel: STAT3 phosphorylation could be inhibited by AG490; B the cells above were also cotransfected with either DN STAT3 or MCSV empty vector as indicated. Luciferase activity was measured and normalized to the Renilla activity. Results are mean \pm SE of three independent experiments performed in triplicate and expressed as the fold induction relative to appropriate control.

would be involved in modulation of the KSHV gene expression. Various KSHV-encoded genes activating STAT3 in KS have been previously described, for instance vGPCR ${ }^{17}$ and vIL-6 have been shown to have transforming activity through activation of the STAT3.$^{25}$ LANA and RTA have been documented to physically interact with STAT3 and activate STAT3. However, LANA induces the dimerization of STAT3 by phosphorylated Tyr $705,{ }^{26}$ whereas RTA does so without affecting tyrosine phosphorylation. ${ }^{20} \mathrm{~V}$ - cyclin $\mathrm{K}$ was found to be associated with the activation domain of STAT3 to inhibit its DNA-binding and transcriptional activities, thus overriding growth suppressive signals through STAT3. ${ }^{3}$ Also, Punjabi et al found that STAT3 activation persists for as long as KSHV latent infection is maintained. ${ }^{27}$ In addition, the JAK/STAT signaling pathway has been described as an important contributor to KSHV replication and increased KSHV viral load by HIV-Tat. ${ }^{28}$
In this study, we found that STAT3 is constitutively activated in KSHV-infected cells. The activity of STAT3 is vGPCRdependent and specifically induced by vGPCR. In this case, tyrosine-phosphorylated STAT3 translocates into the nucleus and activates the transcription of the target VEGF gene by binding to the specific DNA element TT $\left(\mathrm{N}_{4-5}\right)$ AA as measured by pSTAT3-TA-Luc. It should be noted that Wei et al reported a weak or negative expression of STAT3 in normal tissue and strong expression of phosphorylated STAT3 in cancer tissue. ${ }^{29}$ Our data that cells without KSHV infection or with reduced vGPCR expression had weak or negative STAT3 activation support and extrapolate this notion to viral oncogene.

VEGF is ubiquitously found in Kaposi's sarcoma lesions, and is one of the key factors involved in new blood vessel formation within Kaposi's sarcoma tumors. Here, we confirmed previous findings ${ }^{6,8-10}$ that vGPCR could upregulate VEGF in a Kaposi's sarcoma-lesions by using knocking out or knocking 


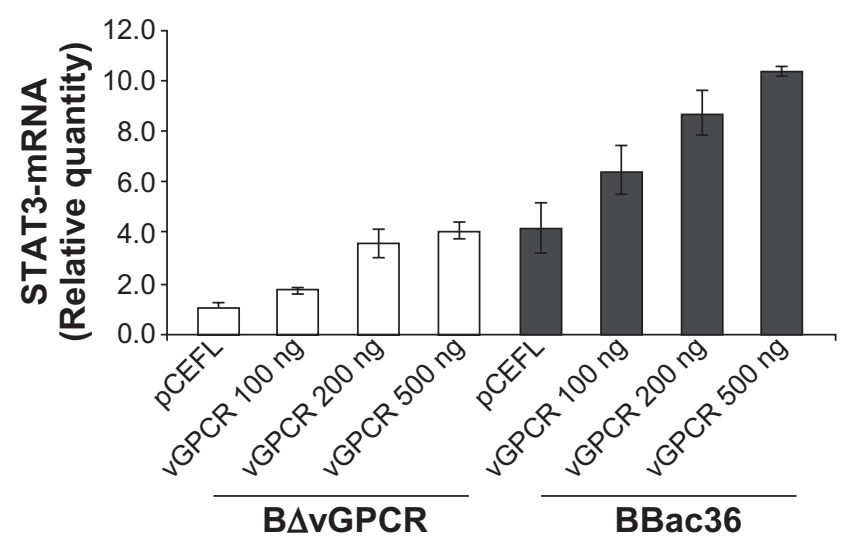

Figure 5 STAT3 mRNA level was elevated by vGPCR. B $\triangle \mathrm{vGPCR}$ and BBac36 cells were transiently transfected with PCEFLHA empty vector and vGPCR plasmid in increasing amounts as indicated. Forty-eight hours after transfection, cells were serum starved for 12 hours. Then total RNA was extracted and used to assay STAT3 mRNA level by quantitative real-time RT-PCR as described in Figure I.

down vGPCR in mECK36-related cells. ${ }^{1}$ More interestingly, treating cells with AG490 or cotransfecting a DN STAT3 specific DNA had a strong inhibitory effect on vGPCR-induced VEGF promoter activity, which suggests that STAT3 is an important mediator in the pathway that connects the viral oncogene to VEGF. Many types of human cancers induced by cellular oncogenes are considered to be associated with STAT3 mediated VEGF expression, some even showing the ability of STAT3 to directly activate the VEGF gene promoter. ${ }^{18,29}$ However, to the best of our knowledge, this is the first evidence that STAT3mediated VEGF expression is induced by the viral oncogene vGPCR.

Since two potential STAT3-binding sites TT $\left(\mathrm{N}_{4-5}\right)$ AA were found in STAT3 promoter, we asked whether STAT3 could stimulate the gene transcription on its own. We found that levels of STAT3 mRNA were elevated as the copies of vGPCR increased in mECK36-related cells. We inferred that this is due to the autoregulation of STAT3. This conclusion is consistent with the previous findings that the STAT3 gene could be autoregulated by STAT3 through its promoter, which contains an STAT3-binding element. ${ }^{30}$ The autoregulation of STAT3 may explain the aggressive behavior of Kaposi's sarcoma lesions at an advanced stage of the disease.

In conclusion, we have demonstrated that vGPCR has broad signaling effects in KS. vGPCR can activate p38-MAP kinase pathways as well as activate STAT3 to induce VEGF expression. According to Marinissen et $\mathrm{al}^{16}$ it is possible to speculate that vGPCR could control activity of p38, and in turn, that of STAT3 transcription factor, which can mediate vGPCR-induced VEGF expression. Undoubtedly, STAT3 is an important mediator in the pathway that connects the viral oncogene vGPCR to VEGF. Based on these findings,
STAT3 could be an effective target for inhibiting tumor VEGF expression and angiogenesis. The data we have described in this study may provide the basis for developing new specific STAT3 targeted drugs for the treatment of advanced Kaposi's sarcoma.

\section{Acknowledgments}

The authors thank the Imaging Core at the University of Miami for providing access to Zeiss LSM 510 confocal microscope and study support. Also Dr. Enrique Mesri for providing the mECK36 cells and critical review of this manuscript.

\section{Disclosure}

The authors report no conflicts of interest in this work.

\section{References}

1. Grisotto MG, Garin A, Martin AP, et al. The human herpesvirus 8 chemokine receptor vGPCR triggers autonomous proliferation of endothelial cells. J Clin Invest. 2006;116(5):1264-1273.

2. Lundquist A, Barre B, Bienvenu F, et al. Kaposi sarcoma-associated viral cyclin $\mathrm{K}$ overrides cell growth inhibition mediated by oncostatin M through STAT3 inhibition. Blood. 2003;101(10):4070-4077.

3. Si H, Robertson ES. Kaposi’s sarcoma-associated herpesvirus-encoded latency-associated nuclear antigen induces chromosomal instability through inhibition of p53 function. J Virol. 2006;80(2):697-709.

4. Taylor JL, Bennett HN, Snyder BA, et al. Transcriptional analysis of latent and inducible Kaposi's sarcoma-associated herpesvirus transcripts in the K4 to K7 region. J Virol. 2005;79(24):15099-15106.

5. Sodhi A, Montaner S, Gutkind JS. Does dysregulated expression of a deregulated viral GPCR trigger Kaposi's sarcomagenesis? Faseb J. 2004; 18(3):422-427.

6. Montaner S, Sodhi A, Ramsdell AK, et al. The Kaposi's sarcomaassociated herpesvirus $\mathrm{G}$ protein-coupled receptor as a therapeutic target for the treatment of Kaposi's sarcoma. Cancer Res. 2006;66(1): 168-174.

7. Mutlu ADA, Cavallin LE, Vincent L, et al. In vivo-restricted and reversible malignancy induced by human herpesvirus-8/KSHV: a cell and animal model of virally induced Kaposi's sarcoma. Cancer Cell. 2007;11:245-258

8. Montaner S, Sodhi A, Molinolo A, et al. Endothelial infection with KSHV genes in vivo reveals that vGPCR initiates Kaposi's sarcomagenesis and can promote the tumorigenic potential of viral latent genes. Cancer Cell. 2003;3(1):23-36.

9. Bais C, Santomasso B, Coso O, et al. G-protein-coupled receptor of Kaposi's sarcoma-associated herpesvirus is a viral oncogene and angiogenesis activator. Nature. 1998;391(6662):86-89.

10. Bais C, van Gellen A, Eroles P, et al. Kaposi's sarcoma associated herpesvirus $\mathrm{G}$ protein-coupled receptor immortalizes human endothelial cells by activation of the VEGF receptor-2/KDR. Cancer Cell. 2003; 3(2):131-143.

11. Cannon M, Cesarman E, Boshoff C. KSHV G protein-coupled receptor inhibits lytic gene transcription in primary-effusion lymphoma cells via p21-mediated inhibition of Cdk2. Blood. 2006;107(1):277-284.

12. Sodhi A, Montaner S, Patel V, et al. The Kaposi's sarcoma-associated herpes virus $\mathrm{G}$ protein-coupled receptor up-regulates vascular endothelial growth factor expression and secretion through mitogen-activated protein kinase and p38 pathways acting on hypoxia-inducible factor 1alpha. Cancer Res. 2000;60(17):4873-4880. 
13. Pati S, Foulke JS Jr, Barabitskaya O, et al. Human herpesvirus 8-encoded vGPCR activates nuclear factor of activated $\mathrm{T}$ cells and collaborates with human immunodeficiency virus type 1 Tat. J Virol. 2003;77(10): 5759-5773.

14. Sgarbanti M, Fiorentini S, Amici C, et al. A requirement for NF-kappaB induction in the production of replication-competent HHV-8 virions. Oncogene. 2004;23(34):5770-5780.

15. Sodhi A, Chaisuparat R, Hu J, et al. The TSC2/mTOR pathway drives endothelial cell transformation induced by the Kaposi's sarcomaassociated herpesvirus G protein-coupled receptor. Cancer Cell. 2006; 10(2):133-143.

16. Marinissen MJ, Tanos T, Bolos M, et al. Inhibition of heme oxygenase-1 interferes with the transforming activity of the Kaposi sarcoma herpesvirus-encoded G protein-coupled receptor. J Biol Chem. 2006; 281(16):11332-11346

17. Burger M, Hartmann T, Burger JA, et al. KSHV-GPCR and CXCR2 transforming capacity and angiogenic responses are mediated through a JAK2-STAT3-dependent pathway. Oncogene. 2005;24(12): 2067-2075.

18. Niu G, Bowman T, Huang M, et al. Roles of activated Src and Stat3 signaling in melanoma tumor cell growth. Oncogene. 2002;21(46): 7001-7010.

19. Faruqi TR, Gomez D, Bustelo XR, et al. Rac1 mediates STAT3 activation by autocrine IL-6. Proc Natl Acad Sci USA. 2001;98(16):9014-9019.

20. Gwack Y, Hwang S, Lim C, et al. Kaposi's sarcoma-associated herpesvirus open reading frame 50 stimulates the transcriptional activity of STAT3. J Biol Chem. 2002;277(8):6438-6442.

21. Hong YK, Foreman K, Shin JW et al. Lymphatic reprogramming of blood vascular endothelium by Kaposi sarcoma-associated herpesvirus. Nat Genet. 2004;36(7):683-685.
22. Lagunoff M, Bechtel J, Venetsanakos AM, et al. De novo infection and serial transmission of Kaposi's sarcoma-associated herpesvirus in cultured endothelial cells. J Virol. 2002;76(5):2440-2448.

23. Naranatt PP, Krishnan HH, Svojanovsky SR, et al. Host gene induction and transcriptional reprogramming in Kaposi's sarcoma-associated herpesvirus (KSHV/HHV-8)-infected endothelial, fibroblast, and B cells: insights into modulation events early during infection. Cancer Res. 2004;64(1):72-84.

24. Wang HW, Trotter MWB, Lagos D, et al. Kaposi sarcoma herpesvirusinduced cellular reprogramming contributes to the lymphatic endothelial gene expression in Kaposi sarcoma. Nat Genet. 2004;36(7):687-693.

25. Hu F, Nicholas, J. Signal transduction by human herpesvirus 8 viral interleukin-6 (vIL-6) is modulated by the nonsignaling gp80 subunit of the IL- 6 receptor complex and is distinct from signaling induced by human IL-6. J Virol. 2006;80(21):10874-10878.

26. Muromoto R, Okabea K, Fujimuro M, et al. Physical and functional interactions between STAT3 and Kaposi's sarcoma-associated herpesvirus-encoded LANA. FEBS Lett. 2006;580(1):93-98.

27. Punjabi AS, Carroll PA, Chen L, et al. Persistent activation of STAT3 by latent KSHV infection of endothelial cells. J Virol. 2006;6:6.

28. Zeng Y, Zhang X, Huang Z, et al. Intracellular tat of human immunodeficiency virus type 1 activates lytic cycle replication of Kaposi's sarcoma-associated herpesvirus: role of the JAK/STAT signaling. J Virol. 2007;81:2401-2417.

29. Wei D, Le X, Zheng L, et al. Stat3 activation regulates the expression of vascular endothelial growth factor and human pancreatic cancer angiogenesis and metastasis. Oncogene. 2003;22(3):319-329.

30. Narimatsu M, Maeda H, Itoh S et al. Tissue-specific autoregulation of the stat 3 gene and its role in interleukin-6-induced survival signals in T cells. Mol Cell Biol. 2001;21(19):6615-6625.
Virus Adaptation and Treatment

\section{Publish your work in this journal}

Virus Adaptation and Treatment is an international, peer-reviewed open access journal focusing on the study of virology, viral adaptation and the development and use of antiviral drugs and vaccines to achieve improved outcomes in infection control and treatment. The journa welcomes original research, basic science, clinical \& epidemiological

\section{Dovepress}

studies, reviews \& evaluations, expert opinion and commentary, case reports and extended reports. The manuscript management system is completely online and includes a very quick and fair peer-review system, which is all easy to use. Visit http://www.dovepress.com/ testimonials.php to read real quotes from published authors. 